\title{
The sleep and circadian problems of Huntington's disease: when, why and their importance
}

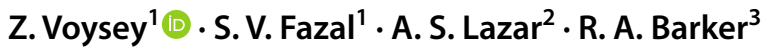

Received: 22 October 2020 / Revised: 15 November 2020 / Accepted: 19 November 2020 / Published online: 23 December 2020

(c) The Author(s) 2020

\begin{abstract}
Introduction Mounting evidence supports the existence of an important feedforward cycle between sleep and neurodegeneration, wherein neurodegenerative diseases cause sleep and circadian abnormalities, which in turn exacerbate and accelerate neurodegeneration. If so, sleep therapies bear important potential to slow progression in these diseases.

Findings This cycle is challenging to study, as its bidirectional nature renders cause difficult to disentangle from effect. Likewise, well-controlled intervention studies are often impractical in the setting of established neurodegenerative disease. It is this that makes understanding sleep and circadian abnormalities in Huntington's disease (HD) important: as a monogenic fully penetrant neurodegenerative condition presenting in midlife, it provides a rare opportunity to study sleep and circadian abnormalities longitudinally, prior to and throughout disease manifestation, and in the absence of confounds rendered by age and comorbidities. It also provides potential to trial sleep therapies at a preclinical or early disease stage. Moreover, its monogenic nature facilitates the development of transgenic animal models through which to run parallel pre-clinical studies. $\mathrm{HD}$, therefore, provides a key model condition through which to gain new insights into the sleep-neurodegeneration interface. Conclusions Here, we begin by summarising contemporary knowledge of sleep abnormalities in HD, and consider how well these parallel those of Alzheimer's and Parkinson's as more common neurodegenerative conditions. We then discuss what is currently known of the sleep-neurodegeneration cyclical relationship in HD. We conclude by outlining key directions of current and future investigation by which to advance the sleep-neurodegeneration field via studies in HD.
\end{abstract}

Keywords Huntington's disease $\cdot$ Sleep $\cdot$ Circadian $\mathrm{rhythm} \cdot$ Neurodegeneration $\cdot$ Dementia

\section{Introduction}

The relationship between sleep and neurodegeneration is a key focus of contemporary neuroscience due to mounting evidence of an important feedforward cycle between the two.

In one arm of the cycle, neurodegenerative conditions beget sleep abnormalities. As a result of a combination of hypothalamic and brainstem dysfunction, along with a

Z. Voysey

zv214@cam.ac.uk

1 Department of Clinical Neurosciences, John Van Geest Centre for Brain Repair, University of Cambridge, Cambridge, UK

2 Faculty of Medicine and Health Sciences, University of East Anglia, Norwich, UK

3 Department of Clinical Neurosciences, John Van Geest Centre for Brain Repair, WT-MRC Cambridge Stem Cell Institute, University of Cambridge, Cambridge, UK loss of long range connectivity, sleep becomes markedly impaired in $40-90 \%$ of patients with neurodegenerative conditions [1-5]. This most commonly manifests as insomnia $[4,6,7]$, but frequently also as circadian rhythm dysfunction [8] or other clinical sleep pathologies such as sleep apnoea or rapid eye movement sleep behaviour disorder (RBD) [4]. Such sleep disruption is a crucial determinant of a patient's quality of life [9], falls risk [10], care giver burden [11] and progression to institutional care [12-15], yet is often not addressed in clinical care.

In the other arm, sleep impairment appears to exacerbate neurodegeneration. Sleep deprivation leads to cognitive deficits in domains including executive function, attention and processing speed, as well as affective disturbances such as impulsivity and emotional lability [16-20]. Sleep abnormalities hence almost certainly contribute to such features common in neurodegenerative conditions. However, increasing evidence contends that abnormal sleep also drives the neurodegenerative pathology directly. For example, sleep 
deprivation has been found to induce neuroinflammation [21] and aberrant protein homeostasis [22] implicated in neurodegenerative pathophysiology. Likewise, slow wave sleep plays a critical role in memory consolidation through synaptic modulation and the translation of hippocampal information to cortical engrams [23-25]. Sleep loss thus also likely contributes to the synaptic dysfunction and memory impairment of many neurodegenerative diseases. Most significant of all has been the discovery of the glymphatic pathway [26]: a glial-lined perivascular network of channels that allows cerebrospinal fluid to drain interstitial brain tissue of waste solutes. The predominant activity of this pathway in slow wave sleep $[27,28]$ has revealed the crucial role sleep plays in clearance of neurotoxic proteins. Both beta-amyloid and tau are now known to be cleared by glymphatic activity $[29,30]$, and sleep deprivation has been consequently been shown to cause marked, rapid increases in these pathogenic protein species [31, 32].

Such findings raise a further question: whether this deleterious feedforward cycle begins with neurodegeneration or abnormal sleep, and thus whether chronic sleep deprivation predisposes to neurodegeneration. In support of the latter, the sleep abnormalities associated with neurodegenerative disease consistently predate symptom onset [7], chronic sleep deprivation has been associated with an almost 50\% increase in risk of dementia in large scale epidemiological studies [33, 34], and seminal studies in chronic sleep disruption among airline staff have suggested a causative relationship $[35,36]$. This is a question of vital importance to public health, given the endemic societal presence of chronic sleep poverty [37].

Understanding this cycle and treating sleep dysfunction may, therefore, present an important means by which to mitigate the severity and progression, or even onset, of neurodegenerative disease.

Yet, the bidirectional nature of the relationship between sleep and neurodegeneration makes it challenging to study, as cause is difficult to disentangle from effect. Moreover, studies are often weakened by age and comorbidity confounds typical in patients with neurodegenerative conditions, and well-controlled intervention studies are often impractical in the setting of established neurodegenerative disease.

It is this that places Huntington's disease (HD) as a critical 'gateway' condition through which to advance our understanding of the sleep-neurodegeneration interface. HD is a fully penetrant, monogenic, autosomal-dominant inherited neurodegenerative condition, caused by a polyglutamine expansion repeat in exon 1 of the huntingtin gene and characterised by a triad of motor, cognitive and psychiatric features typically manifesting between the ages of 35-50. Sleep abnormalities are highly prevalent [38], and, as in other neurodegenerative conditions, precede clinical onset of disease [39]. HD, therefore, provides a crucial opportunity to probe the sleep-neurodegeneration relationship longitudinally, from prior to symptom onset, through the transition phase and into established disease, facilitating a precise interrogation of the strength, timing and directionality of interplay between specific sleep abnormalities and features of neurodegeneration. Moreover, it allows such studies to be undertaken in the absence of age and comorbidity confounds, and offers feasible opportunity to trial therapeutic interventions in prodromal and/or early stage cohorts. Furthermore, its monogenic nature also facilitates the development of transgenic animal models through which to run parallel pre-clinical studies. This is in stark contrast to more common conditions such as Alzheimer's and Parkinson's disease, where (1) typically a premorbid phase can only be recognised in hindsight, (2) a definite diagnosis is challenging to resolve with certainty during life, and (3) transgenic animal models cannot recapitulate the predominant, nonMendelian form of disease.

The study of sleep disturbance in HD, therefore, bears vital potential to inform the wider sleep-neurodegeneration field. Here, we begin by summarising contemporary knowledge of sleep abnormalities in HD, and consider how well these parallel those of Alzheimer's and Parkinson's as more common neurodegenerative conditions. We then discuss what is currently known of the sleep-neurodegeneration cyclical relationship in HD, and the evidence as to how HD causes sleep abnormalities, and whether sleep abnormalities drive HD pathophysiology. We conclude by outlining key directions of current and future investigation by which to advance the sleep-neurodegeneration field via studies in HD.

\section{Sleep and circadian abnormalities in Huntington's disease: evidence to date}

\section{Clinical sleep pathology}

Epidemiological analysis has suggested that clinical sleep pathology is present in as many as $88 \%$ of patients with manifest HD [40]. However, such observations are based on subjective patient reports, and studies comparing these to formal sleep study assessments have revealed prominent discrepancy, both with respect to over- and under-estimation [41, 42]. It is thus judicious to limit any review only to those studies incorporating objective sleep assessments when considering clinical sleep pathologies characterising HD. Of these, the consensus emergent from the literature is of two prominent pathologies: insomnia $[43,44]$ and abnormal motor activity during the sleep period [43, 45-48]. There is controversy as to what this motor activity constitutes [49, 50]: two studies have identified evidence of frequent periodic limb movements during sleep [43, 44], but in addition, several studies have identified complex, high amplitude and 
non-stereotyped movements that occur subsequent to arousals to wakefulness [46-48]. These have been interpreted as chorea [47, 48], repetitive ballistic movements [47], or voluntary repositioning movements rendered atypical by underlying HD-related hypotonia and incoordination [46]. RBD does not appear to be prevalent [43, 44]; nor does sleep disordered breathing $[43,51,52]$ with the exception perhaps of late stage disease [53]. With respect to excessive daytime somnolence, two studies have identified shortened latencies to sleep on objective daytime nap tests $[39,44]$ but the majority of studies have found this to be clinically asymptomatic [43, 44, 54].

In this respect, HD fails to closely recapitulate the sleep abnormalities of other more common neurodegenerative conditions. For example, while insomnia is a common denominator across the spectrum of neurodegeneration, Alzheimer's disease is typified by high rates of sleep disordered breathing [55], while Parkinson's disease is characterised by prominent RBD and excessive daytime somnolence [56]. Nonetheless, such discrepancies are arguably themselves of value, informing how differing patterns and mechanisms of neurodegeneration give rise to divergent sleep pathologies.

\section{Sleep architecture abnormalities}

Sleep is divided into stages by encephalography (EEG), muscle tone and eye movement hallmarks identifiable during polysomnography (sleep studies; PSG). Healthy adult sleep is highly structured, characterised by a transition through progressively deeper non-rapid eye movement sleep (NREM) stages known as N1, N2 and N3/slow wave sleep, followed by rapid eye movement (REM) sleep. This cycle repeats four to five times per night. Aberrations of this structure, referred to as sleep architecture, are highly prevalent in neurodegenerative disease, even in individuals without clinical sleep pathology [42].

There have been numerous clinical PSG studies in HD [39, 42-44, 46, 57], alongside a number in animal models $[58,59]$. There is marked variability among these studies as to the sleep architecture abnormalities that are reported. The reasons for this are likely manifold: a lack of genetic diagnosis in early studies, heterogeneity of clinical stage, medication confounds, use of single night PSG which is vulnerable to habituation artefact, and discrepancy in the PSG features being studied.

However, a recent meta-analysis [60] of these studies has helped to address this. This identified the consistent sleep architecture abnormalities of HD as being those of reduced sleep efficiency, increased time awake after sleep onset, delayed REM sleep onset, and an increased proportion of time spent in the lightest stage of sleep (N1) versus a reduction of time spent in slow wave sleep and REM sleep. Of note, this shares striking similarities with the abnormalities found in Alzheimer's and Parkinson's disease [7], supporting the possible validity of HD as a model for more common conditions with respect to sleep architecture changes.

Cross-sectional studies of premanifest and early stage HD patients have revealed such sleep architecture abnormalities to be present many years prior to manifest disease, and to worsen with approaching manifest disease onset [39, 42, 44]. Among the subsequent manifest HD population, sleep abnormalities appear to continue to progress in parallel with increasing disease severity $[44,57,60]$. Again this mirrors findings in Alzheimer's and Parkinson's [61, 62].

Some studies have also assessed for the presence of sleep fragmentation, rather than simply the total time spent in each sleep stage [39]. This has revealed that fragmentation appears to be an important component of HD sleep architectural disturbance, including in the premanifest phase. Once again this parallels findings in Alzheimer's and Parkinson's, where sleep fragmentation in particular has been identified as a feature of both premonitory and established disease [62-66].

Transgenic HD mouse models $[58,59]$ have corroborated these findings in a number of notable ways, demonstrating increased sleep fragmentation and a loss of slow wave sleep, and progression of sleep abnormalities with disease severity. However, in contrast to human studies, they demonstrate an increase in REM phase. The latter likely reflects imperfect congruity between human and rodent sleep, and between murine HD models (with much longer polyglutamine repeat length and the use of differing promoters) versus human disease.

A handful of studies have employed quantitative EEG: analysis of the relative frequency composition of PSG EEG recordings. Transgenic HD mouse models have demonstrated an increase in gamma frequency content in NREM sleep, and a shift of REM peak frequency from 7 to $6 \mathrm{~Hz}$ [58]. Echoing this, human studies have indicated an increase in gamma content of NREM sleep, and a loss of theta range activity in REM and NREM sleep [39]. Similarly, a recent study employing a technique to localise quantitative EEG changes has also indicated theta component loss: in motor/ premotor areas in NREM, and in limbic regions in REM [41]. Evidence is as yet too sparse to place weight on these findings, but it is of note that theta range activity in sleep has been implicated in neural restoration [67], and a gain in gamma frequencies has been recognised in both schizophrenia and drug-induced psychosis, including in sleep [68].

There is thus relatively comprehensive evidence for sleep architecture changes in HD. Yet, the most informative study has yet to be performed: that of a longitudinal study of these abnormalities in an HD patient cohort, initiated in the premanifest phase and tracked against clinical features. 


\section{Circadian abnormalities}

Circadian abnormalities are also prevalent in HD, with available evidence favouring a loss of circadian amplitude over a defined phase shift. For example, transgenic animal models of HD have demonstrated progressive dampening of circadian rhythmicity, as indicated by diminished diurnal patterns of activity, heart rate variability and body temperature [58, 69, 70]. Clinical studies have supported this, for example demonstrating loss of circadian blood pressure variability [71] and disrupted rest-activity patterns without overt phase shift [59]. Such blunting of circadian rhythm is shared by Alzheimer's and Parkinson's disease, although phase shift appears to also be present in Alzheimer's [8].

Three cross-sectional studies have examined circadian melatonin release in HD patients. These have failed to corroborate one another, reporting mixed results regarding phase shift, reduction or increased temporal spread in melatonin secretion $[54,72,73]$. This lack of consensus owes as much to the paucity of studies as it does to the low number and heterogeneity of participants within them: a large-scale and longitudinal study of melatonin in an HD cohort is, therefore, needed.

\section{How Huntington's disease causes sleep abnormalities}

HD neuropathology is known to affect the hypothalamus $[74,75]$. Since this is the seat of both the body's master circadian clock, the suprachiasmatic nucleus, and orexin neurons responsible for gating sleep/wake states, this is postulated as a key mechanism by which HD brings about sleep and circadian dysfunction. In support of this, abnormalities in orexinergic function have been observed [76, 77] and altered neuronal firing rates have been identified in the suprachiasmatic nucleus in HD animal models [78]. Nonetheless, the applicability of such findings to human disease is questionable. For example, there is poor overlap of hypothalamic abnormalities in HD pre-clinical and clinical studies [79], and clinical imaging studies of hypothalamic changes have shown little correlation with sleep/circadian dysfunction [80].

HD neuropathology is also proposed to bring about sleep dysfunction via influence in other brain regions. For example, HD is recognised to lead to atrophy in brainstem structures such as the locus coeruleus that gate transition between sleep stages $[81,82]$. Likewise, healthy slow wave sleep requires both local cortical and brain-wide synchrony of neuronal oscillatory activity. It may, therefore, also be that the widespread cortical pathology of HD, which affects connectivity even from presymptomatic stages [83], accounts for deficits in slow wave sleep. Moreover, recent evidence has identified a possible role of the striatum in governing arousability during sleep, and the generation of periodic limb movements $[43,84,85]$. As the striatum is a major early and enduring site of HD pathology, this raises a further possible link between HD pathology and sleep disruption. Direct evidence to support such hypotheses is, however, lacking.

Preclinical models have sought to explain circadian disruption in HD through the effect of HD pathology on clock genes. Clock genes are those that interact via intricate feedback loops generating cycles of gene expression with a period of approximately twenty-fours, thereby providing cellular circadian rhythm. These are expressed in the suprachiasmatic nucleus to provide master, coordinating circadian periodicity, but also function in most nucleated cells to provide cell-autonomous circadian rhythm that is then 'tuned' by the master clock and other peripheral cues [86]. Studies of clock genes in transgenic HD mice have almost invariably found evidence of aberrant clock gene expression in vivo, in sites ranging from the suprachiasmatic nucleus to the neocortex to liver $[59,69,87]$. However, significantly, these aberrations resolve upon ex vivo study $[87,88]$. This suggests that circadian dysfunction in HD is driven by system-wide influences on clock gene expression, such as altered melatonin, cortisol or circadian behaviour rhythms, rather than the direct influence of mutant huntingtin protein on intrinsic clock gene function at a cellular level. In support of this, a transgenic sheep model of HD found that circadian behaviour abnormalities dissipated when animals were housed with controls providing normal circadian cueing [89], suggesting that circadian dysfunction is driven more by altered extrinsic zeitgebers than by intrinsic clock gene expression alterations. Nonetheless, such findings are again hypothetical, as there have so far not been any studies of clock genes in HD patients.

\section{Evidence linking Huntington's sleep abnormalities to disease exacerbation}

Most notable of all is the evidence that sleep abnormalities exacerbate HD pathology.

A number of pre-clinical intervention studies have emerged over recent years suggesting that treating sleep abnormalities may improve clinical outcomes in HD. The earliest of these, employing alprazolam in a transgenic HD murine model, indicated that pharmacological imposition of sleep could improve cognitive outcome, independent of anxiolytic effect [88]. A further study by the same group demonstrated that behavioural circadian intervention, mediated by housing animals with intact circadian behaviours, had the same effect [90]. A more recent study of circadian re-entrainment, delivered via blue light therapy in transgenic HD mice, also led to improvements in locomotor activity rhythms and motor performance [91]. 
However, sleep timing or quantity did not alter, and therefore, it is questionable whether the benefit was secondary to the alerting influence of light therapy rather than its impact upon circadian rhythm or sleep quality. Nonetheless, a further study of light-mediated circadian entrainment in transgenic HD mice has demonstrated improved survival rates [92], and another employing timed feeding as a circadian cue has also reported improved motor outcomes [93]. The most recent pre-clinical study of sleep therapy in HD, using an orexin antagonist, has also demonstrated benefit to cognitive outcome [94].

Such studies are of great significance, as they add weight to the notion that sleep abnormalities exacerbate neurodegeneration, and suggest that sleep therapies may, therefore, present a viable means by which to slow disease progression. Given that we currently have no proven disease-modifying treatments for HD, this is of salient importance.

Nevertheless, none of these studies has yet evaluated whether the interventions modified HD outcomes through changes at a pathophysiological level, or whether these were mediated solely through symptomatic gains. A definitive study of whether sleep deprivation promotes accumulation or spread of mutant huntingtin protein, for example, is lacking.

Moreover, to date, there has been no retrospective and only one prospective translational sleep therapy intervention study in HD. This comprised a 9 month pilot study of a multidisciplinary intervention, including exercise, cognitive training, sleep hygiene measures, nutritional guidance and socialisation, in a cohort of sixteen premanifest HD patients [95]. This intervention was well appointed, given that such multi-component therapies carry some of the greatest evidence for efficacy and safety in treating sleep in neurodegenerative conditions [96-99]. Gains in REM sleep were demonstrated, while improvements in slow wave sleep, HD-related structural brain changes and cognitive outcomes were not observed. While disappointing, it should be noted that the study was limited in a number of ways. Besides its small sample size and lack of control group, pre and post intervention PSG was of one-night duration and, therefore, liable to habituation artefact, and cognitive improvement was assessed via only one cognitive test: the Hopkins Verbal Learning Test of memory consolidation. There is, therefore, a prescient need for larger, better controlled and more detailed clinical studies of sleep therapy intervention in HD. These would be enhanced further by the inclusion of biomarkers of disease activity as endpoints. There is growing contemporary evidence for the validity of serum neurofilament light chain, and cerebrospinal tau and mutant huntingtin, in this regard $[100,101]$, making this a natural line of investigation yet to be pursued.

\section{Conclusions and future directions}

Though HD differs from more common conditions like Alzheimer's and Parkinson's with respect to prevalent clinical sleep pathology, there are important parallels evident in relation to the timing and nature of sleep architecture and circadian disturbances. This suggests that HD constitutes a valid model condition, while offering important opportunities not afforded by other neurodegenerative conditions. As such, Huntington's disease is well-placed to provide key new insights into the relationship between sleep and neurodegeneration.

Great advances have been made over the last few years to exploit this potential, in particular in the form of pre-clinical sleep intervention studies examining the link between sleep abnormalities and disease exacerbation. The fact that these have demonstrated improved clinical outcomes in response to treating sleep dysfunction raises important hopes both for HD and the wider neurodegeneration field.

However, a number of salient gaps in the literature remain:

Firstly, there has yet to be a longitudinal clinical study of sleep abnormalities versus clinical outcomes in Huntington's disease, initiated from the premanifest phase. This is a prominent omission, since this is the key opportunity feasible in HD that is not possible in other neurodegenerative conditions. Such a study would enable delineation of the strength and timing of associations between specific sleep abnormalities and features of neurodegeneration, helping to unravel the 'chicken and egg' of the sleep-neurodegeneration relationship. This is a study we are currently completing.

Secondly, there has yet to be a study of clock gene expression in HD patients. The most valuable study would be one designed to differentiate aberrant expression due to altered cell-extrinsic cues from that caused by intrinsic clock dysfunction. This is needed to translate the findings of pre-clinical studies, and to resolve the source of circadian dysfunction to in turn guide therapeutic strategies. Akin to this, more detailed studies of the mechanisms by which HD effects sleep architecture abnormalities would also help guide therapy trials.

Thirdly, studies evaluating the effect of sleep disturbance on HD disease activity, rather than solely clinical features, are currently lacking. This could be achieved, for example, by subjecting transgenic HD mice to sleep deprivation or sleep therapy, and assessing the effect on central nervous system mutant huntingtin accumulation. This is vital to understanding whether sleep abnormalities exacerbate HD disease burden on a pathophysiological or a solely symptomatic level.

Finally, and most importantly, there is a pressing need for robust sleep intervention studies in HD cohorts, to assess whether the promising findings of pre-clinical 
studies translate to patients. Current evidence does not favour the use of traditional sedating agents as sleep interventions in neurodegeneration: studies of antidepressants, antihistamines and antipsychotics in patients with mixed dementia, Alzheimer's and Parkinson's, for example, have suggested that deleterious effects outweigh benefit when used as sleep agents [102-104]. Likewise, there is presently a lack of cogent evidence suggesting significant benefit from modafinil $[105,106]$ or melatonin $[107$, 108]. However, in addition to the described behavioural interventions [109], sodium oxybate [110, 111], orexin antagonists [112,113] and devices facilitating acoustic augmentation of slow wave sleep [114] are all demonstrating potential to improve both sleep and cognitive outcomes in neurodegenerative cohorts, but have yet to be trialled in an HD population. Again, the most valuable studies employing such interventions would be those incorporating biomarkers of disease activity as endpoints, to interrogate effects on clinical features versus pathophysiology.

We believe that such studies represent a research priority, both for Huntington's disease and for informing the wider sleep-neurodegeneration field.

Funding information Roger Barker is supported by the National Institute for Health Research (NIHR) Cambridge Biomedical Research Centre (146281) (the views expressed are those of the author(s) and not necessarily those of the NIHR or the Department of Health and Social Care), and the MRC/WT Stem Cell Institute (203151/Z/16/Z). Zanna Voysey is supported by ABN/Guarantors of Brain Clinical Research Training Fellowship.

\section{Compliance with ethical standards}

Conflicts of interest On behalf of all authors, the corresponding author states that there is no conflict of interest.

Open Access This article is licensed under a Creative Commons Attribution 4.0 International License, which permits use, sharing, adaptation, distribution and reproduction in any medium or format, as long as you give appropriate credit to the original author(s) and the source, provide a link to the Creative Commons licence, and indicate if changes were made. The images or other third party material in this article are included in the article's Creative Commons licence, unless indicated otherwise in a credit line to the material. If material is not included in the article's Creative Commons licence and your intended use is not permitted by statutory regulation or exceeds the permitted use, you will need to obtain permission directly from the copyright holder. To view a copy of this licence, visit http://creativecommons.org/licenses/by/4.0/.

\section{References}

1. Carpenter BD, Strauss M, Patterson MB (1996) Sleep disturbances in community-dwelling patients with Alzheimer's disease. Clin Gerontol 16(2):35-49

2. Barone P et al (2009) The PRIAMO study: a multicenter assessment of nonmotor symptoms and their impact on quality of life in Parkinson's disease. Mov Disord 24(11):1641-1649
3. Tandberg E, Larsen JP, Karlsen K (1998) A community-based study of sleep disorders in patients with Parkinson's disease. Mov Disord 13(6):895-899

4. Guarnieri B et al (2012) Prevalence of sleep disturbances in mild cognitive impairment and dementing disorders: a multicenter Italian clinical cross-sectional study on 431 patients. Dement Geriatr Cogn Disord 33(1):50-58

5. Chahine LM, Amara AW, Videnovic A (2017) A systematic review of the literature on disorders of sleep and wakefulness in Parkinson's disease from 2005 to 2015. Sleep Med Rev 35:33-50 (W.B. Saunders Ltd)

6. Ratti PL et al (2015) Subjective sleep dysfunction and insomnia symptoms in Parkinson's disease: insights from a cross-sectional evaluation of the French CoPark cohort. Park Relat Disord 21(11):1323-1329

7. Malhotra RK (2018) Neurodegenerative disorders and sleep. Sleep Med Clin 13(1):63-70

8. Videnovic A, Lazar AS, Barker RA, Overeem S (2014) The clocks that time us-Circadian rhythms in neurodegenerative disorders. Nat Rev Neurol 10(12):683-693 (Nature Publishing Group)

9. Shafazand $S$ et al (2017) Insomnia, sleep quality, and quality of life in mild to moderate Parkinson's disease. Ann Am Thorac Soc 14(3):412-419

10. Stone KL, Ensrud KE, Ancoli-Israel S (2008) Sleep, insomnia and falls in elderly patients. Sleep Med 9(Suppl): 1

11. Gehrman P, Gooneratne NS, Brewster GS, Richards KC, Karlawish J (2018) Impact of Alzheimer disease patients' sleep disturbances on their caregivers. Geriatr Nurs (Minneap) 39(1):60-65

12. Pollak CP, Perlick D (1991) Sleep problems and institutionalization of the elderly. Top Geriatr 4(4):204-210

13. Pollak CP, Perlick D, Linsner JP, Wenston J, Hsieh F (1990) Sleep problems in the community elderly as predictors of death and nursing home placement. J Community Health 15(2): $123-135$

14. Hope T, Keene J, Gedling K, Fairburn CG, Jacoby R (1998) Predictors of institutionalization for people with dementia living at home with a carer. Int J Geriatr Psychiatry 13(10):682-690

15. Bianchetti A et al (1995) Predictors of mortality and institutionalization in Alzheimer disease patients 1 year after discharge from an Alzheimer dementia unit. Dement Geriatr Cogn Disord 6(2): 108-112

16. Alhola P, Polo-Kantola P (2007) Sleep deprivation: impact on cognitive performance. Neuropsychiatr Dis Treat 3(5):553-567 (Dove Press)

17. Aidman E, Jackson SA, Kleitman S (2019) Effects of sleep deprivation on executive functioning, cognitive abilities, metacognitive confidence, and decision making. Appl Cogn Psychol 33(2):188-200

18. Chua EC-P et al (2014) Sustained attention performance during sleep deprivation associates with instability in behavior and physiologic measures at baseline. Sleep 37(1):27-39

19. Beattie L, Kyle SD, Espie CA, Biello SM (2015) Social interactions, emotion and sleep: a systematic review and research agenda. Sleep Med Rev 24:83-100

20. Lo JC et al (2012) Effects of partial and acute total sleep deprivation on performance across cognitive domains, individuals and circadian phase. PLoS ONE 7(9):e45987

21. Manchanda S, Singh H, Kaur T, Kaur G (2018) Low-grade neuroinflammation due to chronic sleep deprivation results in anxiety and learning and memory impairments. Mol Cell Biochem 449(1-2):63-72

22. Naidoo N, Giang W, Galante RJ, Pack AI (2005) Sleep deprivation induces the unfolded protein response in mouse cerebral cortex. J Neurochem 92(5):1150-1157 
23. Rasch B, Born J (2013) About sleep's role in memory. Physiol Rev 93(2):681-766

24. Todorova R, Zugaro M (2019) Isolated cortical computations during delta waves support memory consolidation. Science 366(6463):377-381

25. Norimoto $\mathrm{H}$ et al (2018) Hippocampal ripples down-regulate synapses. Science 359(6383):1524-1527

26. Rasmussen MK, Mestre H, Nedergaard M (2018) The glymphatic pathway in neurological disorders. Lancet Neurol 17(11):10161024 (Lancet Publishing Group)

27. Xie L et al (2013) Sleep drives metabolite clearance from the adult brain. Science 342(6156):373-377

28. Fultz NE et al (2019) Coupled electrophysiological, hemodynamic, and cerebrospinal fluid oscillations in human sleep. Science 366(6465):628-631

29. Iliff JJ et al (2012) A paravascular pathway facilitates CSF flow through the brain parenchyma and the clearance of interstitial solutes, including amyloid $\beta$. Sci Transl Med 4(147):147ra111

30. Iliff JJ et al (2014) Impairment of glymphatic pathway function promotes tau pathology after traumatic brain injury. J Neurosci 34(49):16180-16193

31. Rothman SM, Herdener N, Frankola KA, Mughal MR, Mattson MP (2013) Chronic mild sleep restriction accentuates contextual memory impairments, and accumulations of cortical A $\beta$ and pTau in a mouse model of Alzheimer's disease. Brain Res 1529:200-208

32. Holth JK et al (2019) The sleep-wake cycle regulates brain interstitial fluid tau in mice and CSF tau in humans. Science 363(6429):80-884

33. Shi L et al (2018) Sleep disturbances increase the risk of dementia: a systematic review and meta-analysis. Sleep Med Rev 40:416 (W.B. Saunders Ltd)

34. Benedict C et al (2015) Self-reported sleep disturbance is associated with Alzheimer's disease risk in men. Alzheimer's Dement 11(9):1090-1097

35. Cho K, Ennaceur A, Cole JC, Suh CK (2000) Chronic jet lag produces cognitive deficits. J Neurosci 20:RC66

36. Cho K (2001) Chronic "jet lag" produces temporal lobe atrophy and spatial cognitive deficits. Nat Neurosci 6(4):567-568

37. Liu Y, Wheaton AG, Chapman DP, Cunningham TJ, Lu H, Croft JB (2016) Prevalence of healthy sleep duration among adults-United States, 2014. MMWR Morb Mortal Wkly Rep 65(6):137-141

38. Herzog-Krzywoszanska R, Krzywoszanski L (2019) Sleep disorders in Huntington's disease. Front Psychiatry 10:221 (Frontiers Media S.A.)

39. Lazar AS et al (2015) Sleep deficits but no metabolic deficits in premanifest Huntington's disease. Ann Neurol 78(4):630-648

40. Taylor N, Bramble D (1997) Sleep disturbance and Huntingdon's disease. Br J Psychiatry 171(4):393-393

41. Piano C et al (2017) Wake and sleep EEG in patients with Huntington disease. Clin EEG Neurosci 48(1):60-71

42. Goodman AOG et al (2011) Asymptomatic sleep abnormalities are a common early feature in patients with Huntington's disease. Curr Neurol Neurosci Rep 11(2):211-217

43. Piano C et al (2015) Polysomnographic findings and clinical correlates in Huntington disease: a cross-sectional cohort study. Sleep 38(9):1489-1495

44. Arnulf I et al (2008) Rapid eye movement sleep disturbances in Huntington disease. Arch Neurol 65(4):482-488

45. Hurelbrink CB, Lewis SJG, Barker RA (2005) The use of the Actiwatch-Neurologica ${ }^{\circledR}$ system to objectively assess the involuntary movements and sleep-wake activity in patients with mildmoderate Huntington's disease. J Neurol 252(6):642-647
46. Neutel D et al (2015) Nocturnal agitation in Huntington disease is caused by arousal-related abnormal movements rather than by rapid eye movement sleep behavior disorder. Sleep Med 16(6):754-759

47. Ranjan S, Kohler S, Harrison MB, Quigg M (2016) Nocturnal post-arousal chorea and repetitive ballistic movement in Huntington's disease. Mov Disord Clin Pract 3(2):200-202

48. Fish DR, Sawyers D, Allen PJ, Blackie JD, Lees AJ, Marsden CD (1991) The effect of sleep on the dyskinetic movements of Parkinson's disease, Gilles De La Tourette syndrome, Huntington's disease, and Torsion dystonia. Arch Neurol 48(2):210-214

49. Piano C, Bentivoglio AR, Cortelli P, Della Marca G (2016) Motor-related sleep disorders in Huntington disease. A comment on Neute et al.: "nocturnal agitation in Huntington disease is caused by arousal-related abnormal movements rather than by rapid eye movement sleep behavior disorder" by Neutel et al. Sleep Med 20:172-173 (Elsevier B.V.)

50. Neutel D, Leu-Semenescu S, Roze E, Arnulf I (2016) Reply to Piano et al. Sleep Med 20:174-175 (Elsevier B.V.)

51. Bollen EL et al (1988) Respiration during sleep in Huntington's chorea. J Neurol Sci 84(1):63-68

52. Cuturic M, Abramson RK, Vallini D, Frank EM, Shamsnia M (2009) Sleep patterns in patients with Huntington's disease and their unaffected first-degree relatives: a brief report. Behav Sleep Med 7(4):245-254

53. Antczak J et al (2013) Huntington's disease and sleep related breathing disorders. Hygiea Public Health 48(4):449-455

54. Aziz NA, Anguelova GV, Marinus J, Lammers GJ, Roos RAC (2010) Sleep and circadian rhythm alterations correlate with depression and cognitive impairment in Huntington's disease. Park Relat Disord 16(5):345-350

55. Ancoli-Israel S, Kripke DF, Klauber MR, Mason WJ, Fell R, Kaplan O (1991) Sleep-disordered breathing in communitydwelling elderly. Sleep 14(6):486-495

56. Loddo G et al (2017) The treatment of sleep disorders in Parkinson's disease: from research to clinical practice. Front Neurol $8(42): 1-18$

57. Wiegand M, Möller AA, Schreiber W, Lauer C, Krieg JC (1991) Brain morphology and sleep EEG in patients with Huntington's disease. Eur Arch Psychiatry Clin Neurosci 240(3):148-152

58. Kantor S, Szabo L, Varga J, Cuesta M, Morton AJ (2013) Progressive sleep and electroencephalogram changes in mice carrying the Huntington's disease mutation. Brain 136(7):2147-2158

59. Morton AJ, Wood NI, Hastings MH, Hurelbrink C, Barker RA, Maywood ES (2005) Disintegration of the sleep-wake cycle and circadian timing in Huntington's disease. J Neurosci 25(1):157-163

60. Zhang Y et al (2019) Sleep in Huntington's disease: a systematic review and meta-analysis of polysomongraphic findings. Sleep 42(10):zsz154

61. Prinz PN et al (1982) Sleep, EEG and mental function changes in senile dementia of the Alzheimer's type. Neurobiol Aging 3(4):361-370

62. Jasti DB, Mallipeddi S, Apparao A, Vengamma B, Kolli S, Mohan A (2018) Quality of sleep and sleep disorders in patients with parkinsonism: a polysomnography based study from rural South India. J Neurosci Rural Pract 9(1):92-99

63. Peter-Derex L, Yammine P, Bastuji H, Croisile B (2015) Sleep and Alzheimer's disease. Sleep Med Rev 19:29-38 (W.B. Saunders Ltd)

64. Cai GE, Luo S, Chen LN, Lu JP, Huang YJ, Ye QY (2019) Sleep fragmentation as an important clinical characteristic of sleep disorders in Parkinson's disease: a preliminary study. Chin Med J (Engl) 132(15):1788-1795 
65. Lim ASP, Kowgier M, Yu L, Buchman AS, Bennett DA (2013) Sleep fragmentation and the risk of incident Alzheimer's disease and cognitive decline in older persons. Sleep 36(7):1027-1032

66. Sohail S, Yu L, Schneider JA, Bennett DA, Buchman AS, Lim ASP (2017) Sleep fragmentation and Parkinson's disease pathology in older adults without Parkinson's disease. Mov Disord 32(12):1729-1737

67. Feinberg I, Campbell IG (2013) Longitudinal sleep EEG trajectories indicate complex patterns of adolescent brain maturation. Am J Physiol-Regul Integ Comp Physiol 304(4):296

68. Herrmann CS, Demiralp T (2005) Human EEG gamma oscillations in neuropsychiatric disorders. Clin Neurophysiol 116(12):2719-2733

69. Kudo T et al (2011) Dysfunctions in circadian behavior and physiology in mouse models of Huntington's disease. Exp Neurol 228(1):80-90

70. Rudenko O, Tkach V, Berezin V, Bock E (2009) Detection of early behavioral markers of Huntington's disease in R6/2 mice employing an automated social home cage. Behav Brain Res 203(2):188-199

71. Bellosta Diago E et al (2017) Circadian rhythm and autonomic dysfunction in presymptomatic and early Huntington's disease. Park Relat Disord 44:95-100

72. Kalliolia E et al (2014) Plasma melatonin is reduced in Huntington's disease. Mov Disord 29(12):1511-1515

73. Alders J, Smits M, Kremer B, Naarding P (2009) The role of melatonin in sleep disturbances in end-stage Huntington's disease. J Neuropsychiatry Clin Neurosci 21(2):226-227 (American Psychiatric Publishing Inc)

74. Politis M, Pavese N, Tai YF, Tabrizi SJ, Barker RA, Piccini P (2008) Hypothalamic involvement in Huntington's disease: an in vivo PET study. Brain 131(11):2860-2869

75. Petersén D, Gabery S (2012) Hypothalamic and limbic system changes in Huntington's diseaseAsa. J Huntingtons Dis 1:5-16

76. Peterseén $\AA$ et al (2005) Orexin loss in Huntington's disease. Hum Mol Genet 14(1):39-47

77. Aziz A et al (2008) Hypocretin and melanin-concentrating hormone in patients with Huntington disease. Brain Pathol 18(4):474-483

78. Kuljis D, Kudo T, Tahara Y, Ghiani CA, Colwell CS (2018) Pathophysiology in the suprachiasmatic nucleus in mouse models of Huntington's disease. J Neurosci Res 96(12):1862-1875

79. van Wamelen DJ, Aziz NA, Roos RAC, Swaab DF (2014) Hypothalamic alterations in Huntington's disease patients: comparison with genetic rodent models. J Neuroendocrinol 26(11):761-775

80. Bartlett DM et al (2019) Investigating the relationships between hypothalamic volume and measures of circadian rhythm and habitual sleep in premanifest Huntington's disease. Neurobiol Sleep Circadian Rhythm 6:1-8

81. Rosas HD et al (2003) Evidence for more widespread cerebral pathology in early HD: an MRI-based morphometric analysis. Neurology 60(10):1615-1620

82. Zweig RM et al (1992) Locus Coeruleus involvement in Huntington's disease. Arch Neurol 49(2):152-156

83. Pini L et al (2020) Aberrant brain network connectivity in presymptomatic and manifest Huntington's disease: a systematic review. Hum Brain Mapp 41(1):256-269

84. Mena-Segovia J, Cintra L, Prospéro-García O, Giordano M (2002) Changes in sleep-waking cycle after striatal excitotoxic lesions. Behav Brain Res 136(2):475-481

85. Stoffers D et al (2014) The caudate: a key node in the neuronal network imbalance of insomnia? Brain 137(2):610-620

86. Cox KH, Takahashi JS (2019) Circadian clock genes and the transcriptional architecture of the clock mechanism. J Mol Endocrinol 63(4):R93-R102
87. Maywood ES et al (2010) Disruption of peripheral circadian timekeeping in a mouse model of Huntington's disease and its restoration by temporally scheduled feeding. J Neurosci 30(30):10199-10204

88. Pallier PN et al (2007) Pharmacological imposition of sleep slows cognitive decline and reverses dysregulation of circadian gene expression in a transgenic mouse model of Huntington's disease. J Neurosci 27(29):7869-7878

89. Jennifer Morton A et al (2014) Early and progressive circadian abnormalities in Huntington's disease sheep are unmasked by social environment. Hum Mol Genet 23(13):3375-3383

90. Pallier PN, Morton AJ (2009) Management of sleep/wake cycles improves cognitive function in a transgenic mouse model of Huntington's disease. Brain Res 1279:90-98

91. Bin Wang $\mathrm{H}$ et al (2017) Blue light therapy improves circadian dysfunction as well as motor symptoms in two mouse models of Huntington's disease. Neurobiol Sleep Circadian Rhythm. 2:39-52

92. Ouk K, Aungier J, Morton AJ (2017) Prolonged day length exposure improves circadian deficits and survival in a transgenic mouse model of Huntington's disease. Neurobiol Sleep Circadian Rhythm 2:27-38

93. Whittaker DS et al (2018) Circadian-based treatment strategy effective in the BACHD mouse model of Huntington's disease. J Biol Rhythms 33(5):535-554

94. Cabanas M et al (2019) Neurophysiological and behavioral effects of anti-orexinergic treatments in a mouse model of Huntington's disease. Neurotherapeutics 16(3):784-796

95. Bartlett DM et al (2019) Effect of multidisciplinary rehabilitation on sleep outcomes in individuals with preclinical Huntington disease: an exploratory study. Ann Phys Rehabil Med 63:570-573

96. Richards KC, Beck C, O'Sullivan PS, Shue VM (2005) Effect of individualized social activity on sleep in nursing home residents with dementia. J Am Geriatr Soc 53(9):1510-1517

97. Connell BR, Sanford JA, and Lewis D (2008) Therapeutic effects of an outdoor activity program on nursing home residents with dementia. In: Outdoor environments for people with dementia, vol 21, no 3-4. Taylor and Francis, pp 194-209

98. Naylor E et al (2000) Daily social and physical activity increases slow-wave sleep and daytime neuropsychological performance in the elderly. Sleep 23(1):87-95

99. Richards KC et al (2011) Strength training, walking, and social activity improve sleep in nursing home and assisted living residents: randomized controlled trial. J Am Geriatr Soc 59(2):214-223

100. Rodrigues FB et al (2016) Cerebrospinal fluid total tau concentration predicts clinical phenotype in Huntington's disease. J Neurochem 139(1):22-25

101. Byrne LM et al (2018) Evaluation of mutant huntingtin and neurofilament proteins as potential markers in Huntington's disease. Sci Transl Med 10(458):eaat7108

102. Ellul $\mathrm{J}$ et al (2007) The effects of commonly prescribed drugs in patients with Alzheimer's disease on the rate or deterioration. J Neurol Neurosurg Psychiatry 78(3):233-239

103. Scoralick FM, Louzada LL, Quintas JL, Naves JOS, Camargos EF, Nóbrega OT (2017) Mirtazapine does not improve sleep disorders in Alzheimer's disease: results from a double-blind, placebo-controlled pilot study. Psychogeriatrics 17(2):89-96

104. Mittal V, Kurup L, Williamson D, Muralee S, Tampi RR (2011) Risk of cerebrovascular adverse events and death in elderly patients with dementia when treated with antipsychotic medications: a literature review of evidence. Am J Alzheimer's Dis Other Demen 26(1):10-28 (SAGE Publications Inc)

105. Frakey LL, Salloway S, Buelow M, Malloy P (2012) A randomized, double-blind, placebo-controlled trial of modafinil for 
the treatment of apathy in individuals with mild-to-moderate Alzheimer's disease. J Clin Psychiatry 73(6):796-801

106. Högl B et al (2002) Modafinil for the treatment of daytime sleepiness in Parkinson's disease: a double-blind, randomized, crossover, placebo-controlled polygraphic trial. Sleep 25(8):905-909

107. Zhang W et al (2016) Exogenous melatonin for sleep disorders in neurodegenerative diseases: a meta-analysis of randomized clinical trials. Neurol Sci 37(1):57-65

108. Mccleery J, Cohen DA, Sharpley AL (2016) Pharmacotherapies for sleep disturbances in dementia. Cochrane Database Syst Rev 2016(11):CD009178

109. Cassidy-Eagle E, Siebern A, Unti L, Glassman J, O'Hara R (2018) Neuropsychological functioning in older adults with mild cognitive impairment and insomnia randomized to CBT-I or control group. Clin Gerontol 41(2):136-144

110. Walsh JK et al (2010) Enhancing slow wave sleep with sodium oxybate reduces the behavioral and physiological impact of sleep loss. Sleep 33(9):1217-1225
111. Büchele F et al (2018) Sodium oxybate for excessive daytime sleepiness and sleep disturbance in Parkinson disease: a randomized clinical trial. JAMA Neurol 75(1):114-118

112. Herring WJ et al (2020) Polysomnographic assessment of suvorexant in patients with probable Alzheimer's disease dementia and insomnia: a randomized trial. Alzheimer's Dement 16(3):541-551

113. Neylan TC et al (2020) Acute cognitive effects of the hypocretin receptor antagonist almorexant relative to zolpidem and placebo: a randomized clinical trial. Sleep 43(10):zsaa080

114. Papalambros NA et al (2019) Acoustic enhancement of sleep slow oscillations in mild cognitive impairment. Ann Clin Transl Neurol 6(7):1191-1201 